\title{
Reception of Radio Waves from Pulsars
}

\author{
R. T. Gangadhara \\ Indian Institute of Astrophysics, Bangalore - 560034, India
}

\begin{abstract}
The beamed emission by relativistic sources moving along the magnetic dipolar field lines occurs in the direction of tangents to the field lines. To receive such a beamed radiation, the line of sight must align with the tangent within the beaming angle $1 / \gamma$, where $\gamma$ is the particle Lorentz factor. By solving the viewing geometry, in an inclined and rotating dipole magnetic field, we show that at any given pulse phase the observer can receive the radiation only from specific altitudes. We find that the outer conal emission is received from higher altitudes than the inner conal components including the core. At any pulse phase, low frequency emission comes from the higher altitudes than higher-frequency emission. As an application of our model, we have used it to explain the emission heights of conal components in PSR B0329+54.
\end{abstract}

\section{Introduction}

Attempts have been made to interpret pulsar radio beams in terms of emission in a purely dipolar magnetic field. Gangadhara \& Gupta (2001) have estimated the emission heights of different radio pulse components in PSR B0329+54 based on the aberration-retardation phase shift, and the revised estimates are given by Dyks, Rudak \& Harding (2003). Here we solve the viewing geometry and estimate the altitudes from which the observer can receive the radio waves.

\section{Emission Beam Geometry}

Consider a magnetic dipole situated at the origin with magnetic axis $(\hat{\mathbf{m}})$ inclined by $\alpha$ with respect to the rotation axis $(\hat{\boldsymbol{\Omega}})$, and rotated by $\phi^{\prime}$ around $\hat{\boldsymbol{\Omega}}$. Let $\hat{\mathbf{n}}=(\sin \zeta, 0, \cos \zeta)$ be the line of sight, where $\zeta=\alpha+\beta$, and $\beta$ is the line-of-sight impact parameter.

In a relativistic flow, the emitted radiation is beamed in the direction of field line tangent $\hat{\mathbf{b}}$, so at any instant the observed radiation comes from a spot in the magnetosphere where the tangent vector points in the direction $\hat{\mathbf{n}}$ of the observer. To receive such radiation, the semi opening angle of the emission beam $\Gamma=\arccos (\hat{\mathbf{n}} \cdot \hat{\mathbf{m}})$ must be approximately equal to the opening angle of the field lines $\tau=\arccos (\hat{\mathbf{b}} \cdot \hat{\mathbf{m}})$. Therefore, the magnetic colatitude $(\theta)$ is given by

$$
\cos (2 \theta)=\frac{1}{3}\left(\cos \Gamma \sqrt{8+\cos ^{2} \Gamma}-\sin ^{2} \Gamma\right), \quad-\pi \leq \Gamma \leq \pi .
$$



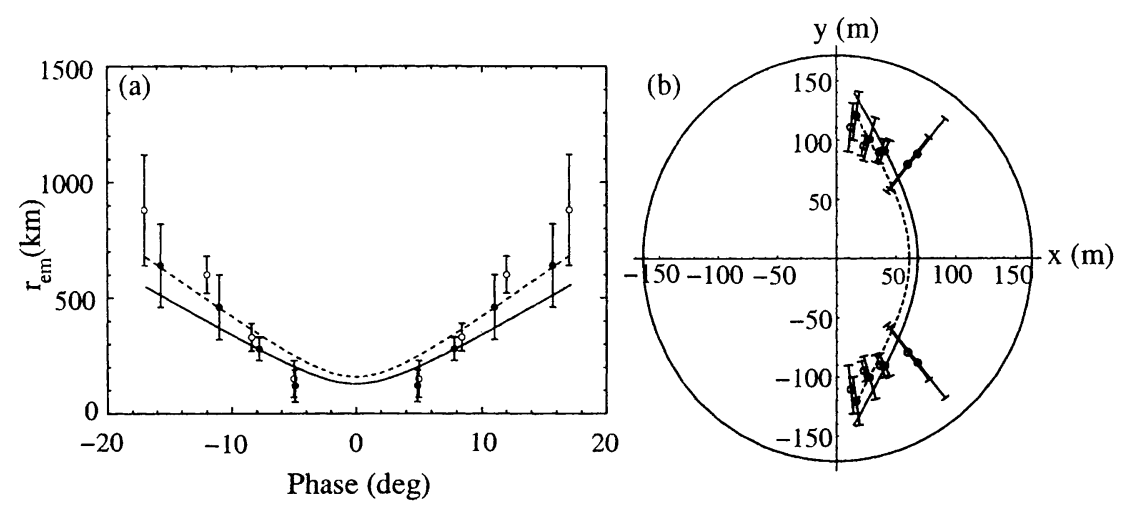

Figure 1. (a) Emission heights in PSR B0329+54: solid and dashed line curves are for the emissions at $606 \mathrm{MHz}$ and $325 \mathrm{MHz}$, respectively. The emission heights estimated from aberration-retardation phase shift are superposed: the points are marked with $\circ$ for $325 \mathrm{MHz}$ and $\bullet$ for 606 $\mathrm{MHz}$. (b) Polar cap with field lines associated with foot of emission.

Next, the magnetic azimuth $(\phi)$ of the emission point can be obtained by finding $\hat{\mathbf{b}}$ which is parallel to $\hat{\mathbf{n}}$ :

$$
\sin \phi=-\sin \zeta \sin \phi^{\prime} \csc \Gamma .
$$

For $\beta>0$, on the leading side the maximum value for $\phi$ allowed by the viewing geometry is $\pi / 2$, which in turn allows to find the maximum pulse window $W=$ $2 \phi^{\prime}$, where $\phi^{\prime}$ is the pulse phase at which $\phi$ approaches $\pi / 2$. Using $\alpha=30^{\circ}$ and $\beta=2.1^{\circ}$ for PSR B0329+54, we find $\theta_{\max } \sim 8^{\circ}, \Gamma_{\max } \sim 12^{\circ}$ and $W \sim 46^{\circ}$.

Pulsar radio emission is generally believed to be coherent curvature radiation by a secondary pair plasma streaming along the dipolar magnetic field lines. The curvature emission peaks at the characteristic frequency (e.g., Eq. [45] of Ruderman \& Sutherland 1975). For a given frequency and a Lorentz factor $\gamma$, we can estimate the radius of curvature $\rho$, which in turn allows to find the field line constant. So, using $\gamma=340$ and 390 we estimated the emission height (see, Fig. 1a) of radiation at $325 \mathrm{MHz}$ and $606 \mathrm{MHz}$, respectively. On the other hand, by accepting the emission heights derived from the aberration-retardation phase shift, we estimated $\gamma$ and $\rho$ expected: $0.15 \leq \rho / r_{\mathrm{LC}} \leq 0.33,286 \leq \gamma \leq 370$ for $325 \mathrm{MHz}$ emission, and $0.12 \leq \rho / r_{\mathrm{LC}} \leq 0.26,328 \leq \gamma \leq 420$ for $606 \mathrm{MHz}$.

The polar cap with field lines associated with emission is given in Figure 1b, where the $z$-axis is chosen to be parallel to $\hat{\mathbf{m}}$ and $\mathrm{x}$ to lie in the $\hat{\boldsymbol{\Omega}}$ - $\hat{\mathbf{m}}$ plane. It is nearly elliptical with radius of $164 \mathrm{~m}$ and $171 \mathrm{~m}$ in the $\mathrm{x}$ and $\mathrm{y}$ directions.

\section{References}

Gangadhara, R. T., \& Gupta, Y. 2001, ApJ, 555, 31

Dyks, J., Rudak, B., \& Harding, A. K. 2003, ApJ, in press (astro-ph/0307251)

Ruderman, M. A., \& Sutherland, P. G. 1975, ApJ, 196, 51 